Article

\title{
Outage Analysis of Relay-Assisted NOMA in Cooperative Cognitive Radio Networks with SWIPT
}

\author{
Kun Tang $(1)$ and Shaowei Liao * \\ Guangdong Provincial Key Laboratory of Millimeter-Wave and Terahertz, School of Electronic and Information \\ Engineering, South China University of Technology, Guangzhou 510641, China; tangkun@scut.edu.cn \\ * Correspondence: liaoshaowei@scut.edu.cn
}

Received: 22 September 2020; Accepted: 24 October 2020; Published: 26 October 2020

\begin{abstract}
In this paper, we investigate a relay-assisted cooperative spectrum sharing for the considered non-orthogonal multiple access (NOMA) scheme in cognitive radio networks, where the relay node assists the base station (BS) to transmit the superimposed composite signal to two receivers by utilizing an amplified-and-forward (AF) technique with simultaneous wireless information and power transfer (SWIPT). The exact expressions for outage probabilities of two receivers are derived in closed forms. Moreover, a joint optimization of power allocation and the proportion of information splitting for energy harvesting is proposed in terms of energy efficiency (EE) maximization under required data reliability. Simulation results validate the analytical results since the analytical results match well with simulation results and demonstrate the performance advantages of the proposed scheme over other schemes and direct transmission.
\end{abstract}

Keywords: relay-assisted NOMA; simultaneous wireless information and power transfer; amplified-and-forward relaying; outage probability; energy efficiency optimization

\section{Introduction}

Non-orthogonal multiple access (NOMA) has been regarded as a promising multiple access technique for supporting massive machine-type communication scenarios due to its higher spectral efficiency for ultra-dense network deployment [1,2]. The NOMA scheme is based on assigning higher power to users with worse channel conditions, which will lead the users in cell-edge to get more energy. Unlike conventional orthogonal multiple access (OMA) scheme, NOMA techniques can effectively utilize same resource blocks such as time, frequency, and power, to implement multiple-access applications, which could effectively improve the system transmission performance [3]. Therefore, NOMA can provide massive connectivity, low latency, as well as high spectral efficiency and reliability compared to OMA. However, the inter-cell interference is more severe in NOMA compared to OMA due to biased power allocation toward users in cell-edge.

In addition, the improvement of the spectral sharing were widely studied in cognitive radio (CR) networks, where the secondary users can either access the licensed spectrum belonging to primary users without causing interference or opportunistically assist the data transmission of the primary users in exchange for the spectrum sharing $[4,5]$. In practice, simultaneous wireless information and power transfer (SWIPT) has emerged recently for low-power electronic devices, which can harvest energy and process the information from radio frequency (RF) signals concurrently [6,7].

In the context of CR, the NOMA techniques have been investigated in [8-10]. A cooperative transmission scheme aimed at exploiting the inherent spatial diversity of CR-NOMA system was proposed for the application of downlink transmission [8]. The authors in [9] integrated the NOMA technique into cognitive orthogonal frequency-division multiplexing (OFDM) systems. An alternate iteration framework was proposed to jointly optimize the parameters, i.e., sensing duration, 
user scheduling, and power allocation, for maximizing the capacity of the system. A dual-hop underlay CR-NOMA network was considered and the outage probability of the end-to-end was analyzed as performance metric [10]. Furthermore, the reliability and security performance of cooperative NOMA in CR networks was evaluated by developing a tractable analysis framework [11]. Recently, combining NOMA with wireless-powered communication (WPC) networks has received a large amount of attention. In [12], NOMA for the SWIPT system with two energy harvesting users was investigated and the boundary of the rate region for the downlink was then derived. The authors in [13] proposed a cooperative SWIPT-based NOMA protocol in multiple-input single-output (MISO) systems, where the optimal beamforming and power splitting has been analyzed by utilizing equivalent transform with semidefinite relaxation technique. Moreover, the error rate of relay-assisted NOMA networks with SWIPT technique was investigated and the exact expression of pairwise error probability was derived [14]. The EE optimization for CR-NOMA systems were also analyzed in [15] and [16]. In [15], an EE optimization problem with satisfying quality of service (QoS) for all users was considered in a CR-NOMA network. In order to solve the non-convex optimization problem, an algorithm based on Sequential Convex Approximation (SCA) method was then developed. For the SWIPT-based CR-NOMA systems, the authors in [16] analyzed the EE optimization in both overlay network and underlay network with corresponding transmission protocols. A cooperative NOMA scheme with SWITP was proposed in underlay CR networks [17], where a relay first harvested energy from the secondary transmitter based on power splitting scheme and then cooperatively forwarded the encoded signals to two receivers.

In [17], the signal transmitting can only depend on the relay node when it can harvest energy and correctly receive both signals for two receivers, i.e., the whole transmission process might be in outage when the relay node failed to decode the received signals. In contrast with [17], we consider the scenario whereby the signals can be directly transmitted to the receivers in the first phase while the relay node performs energy harvesting, then the relay node assists the signals' transmission to receivers with AF scheme in the second phase. The main contributions of this work can be summarized as follows.

- We develop a wireless energy harvesting and signal transmission protocol for the considered non-orthogonal multiple access (NOMA) scheme in cognitive radio networks. In particular, the whole transmission block is divided into two phases. During the first phase, the base station (BS) transmits a superposed signal to receivers while the relay node harvests energy and obtains data from the received signal. In the second phase, the relay node forwards the BS's signals by using the harvested energy.

- The exact expressions for the outage probabilities of both the receivers are derived and the analytical results are validated through Monte Carlo simulations, which verifies the correctness of our analyses.

- By considering the maximization of the average EE under the required transmission rates, we propose a full-search algorithm to obtain the optimal power allocation and proportion of information splitting coefficients, which provides a practical guideline that the optimized coefficients enable the proposed protocol to achieve a better performance of EE compared with other scheme and direct transmission.

The rest of this paper can be organized as follows: Section 2 illustrates the system model and transmission protocol. In Section 3, exact outage probabilities for both receivers are derived, and parameter optimization is analyzed. Section 4 contains simulation results and the conclusion of this paper is summarized in Section 5 .

\section{System Model}

We consider a relay-assisted NOMA scheme with SWIPT in cooperative cognitive radio networks, as shown in Figure 1, where a BS provides information transmission service to two receivers (U1 and 
U2) with the assistance of relay node R, which harvests energy from the BS. With the execution of NOMA scheme, more transmit power of BS is allocated for far users, while less transmit power for near users. Each node is equipped with a single antenna operating in half-duplex mode. All channels are assumed to undergo the quasi-static Rayleigh fading channel. Let $h_{i j}$ denote the channel from node $i$ to node $j$ and the channel power gain is expressed as $\left|h_{i j}\right|^{2}$, which follows exponentially distributed with mean $\lambda_{i j}=d_{i j}^{-\theta}$, where $d_{i j}$ and $\theta$ denote the distance between nodes $i$ and $j$, and pass-loss exponent, respectively $[18,19]$.

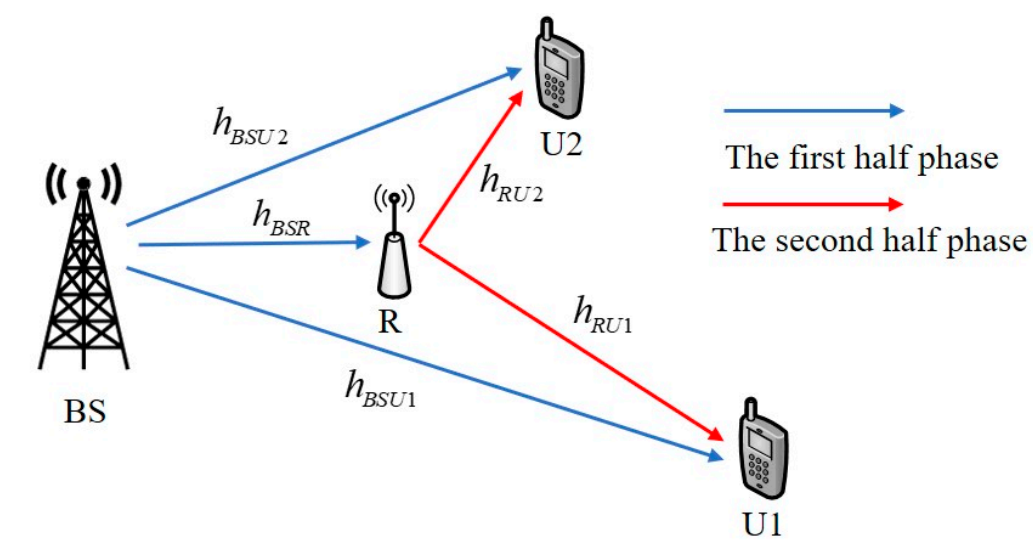

Figure 1. The illustration of relay-assisted NOMA scheme with SWIPT in cooperative cognitive radio network.

The whole operation includes two equal phases. In the first phase, BS transmits the superpositioncoded signal $x_{B S}=\sqrt{\alpha_{1}} x_{1}+\sqrt{\alpha_{2}} x_{2}$ to nodes $\mathrm{R}, \mathrm{U} 1$, and $\mathrm{U} 2$, where $x_{1}$ and $x_{2}$ are the desired signals of $\mathrm{U} 1$ and $\mathrm{U} 2$, respectively, and power allocation coefficients are comprised as $\alpha_{1}$ and $\alpha_{2}$ with $\alpha_{1}+\alpha_{2}=1$ $\left(\alpha_{1}>\alpha_{2}\right)$. Thus, the received signals at the nodes can be expressed as

$$
\begin{gathered}
y_{R}=\sqrt{P_{B S}} h_{B S R}\left(\sqrt{\alpha_{1}} x_{1}+\sqrt{\alpha_{2}} x_{2}\right)+n_{R}, \\
y_{U 1}^{\mathrm{I}}=\sqrt{P_{B S}} h_{B S U 1}\left(\sqrt{\alpha_{1}} x_{1}+\sqrt{\alpha_{2}} x_{2}\right)+n_{U 1}, \\
y_{U 2}^{\mathrm{I}}=\sqrt{P_{B S}} h_{B S U 2}\left(\sqrt{\alpha_{1}} x_{1}+\sqrt{\alpha_{2}} x_{2}\right)+n_{U 2},
\end{gathered}
$$

respectively, where $P_{B S}$ is the transmit power of the $\mathrm{BS}, n_{i} \sim C N\left(0, \delta_{i}^{2}\right)(i=R, S U 1, S U 2)$ denotes the additive white Gaussian noise (AWGN) at nodes. Based on the power splitting method, the received signal at the node $\mathrm{R}$ can be divided into two streams, one for energy harvesting and the other for information transfer. The observation for energy harvesting of $\mathrm{R}$ is given by

$$
\sqrt{\beta} y_{R}=\sqrt{\beta P_{B S}} h_{B S R}\left(\sqrt{\alpha_{1}} x_{1}+\sqrt{\alpha_{2}} x_{2}\right)+\sqrt{\beta} n_{R}
$$

where $\beta \in(0,1)$ denotes the portion of information split for energy harvesting. Then, the transmit power of relay node $R$ can be calculated as

$$
P_{R}=\eta \beta P_{B S}\left|h_{B S R}\right|^{2}
$$

Correspondingly, the received SINR in the first phase at $\mathrm{U} 1$ is given by

$$
\gamma_{U 1}^{\mathrm{I}}=\frac{\alpha_{1} P_{B S}\left|h_{B S U 1}\right|^{2}}{\alpha_{2} P_{B S}\left|h_{B S U 1}\right|^{2}+\delta_{U 1}^{2}} .
$$


The SIC technique is applied at the U2, which first detects the signal of U1 and then obtains its desired signal. Therefore, the SINRs for the U2 to detect both the signals of $\mathrm{U} 1$ and $\mathrm{U} 2$ are respectively expressed as

$$
\gamma_{U 2,1}^{\mathrm{I}}=\frac{\alpha_{1} P_{B S}\left|h_{B S U 2}\right|^{2}}{\alpha_{2} P_{B S}\left|h_{B S U 2}\right|^{2}+\delta_{S U 2}^{2}}, \gamma_{U 2,2}^{\mathrm{I}}=\frac{\alpha_{2} P_{B S}\left|h_{B S U 2}\right|^{2}}{\delta_{B S U 2}^{2}} .
$$

During the second phase, the relay node $\mathrm{R}$ forwards the residual signal $\sqrt{1-\beta} y_{R}$ based on the AF scheme. Thus, the broadcasting information at the relay node $\mathrm{R}$ can be written as

$$
x_{R}=G\left(\sqrt{1-\beta} y_{R}+n_{b}\right),
$$

where $n_{b} \sim C N\left(0, \delta_{b}^{2}\right)$ denotes the AWGN introduced by the signal conversion from passband to baseband at the node $R$, the power normalization factor $G$ is given by

$$
G=\frac{1}{\sqrt{(1-\beta)\left(P_{B S}\left|h_{B S R}\right|^{2}+\delta_{R}^{2}\right)+\delta_{b}^{2}}} \approx \frac{1}{\sqrt{(1-\beta) P_{B S}\left|h_{B S R}\right|^{2}}} .
$$

Note that $\delta_{R}^{2}$ is much smaller than the noise power introduced by the baseband circuit. Moreover, compared with the $(1-\beta) P_{B S}\left|h_{B S R}\right|^{2}, \delta_{b}^{2}$ can be neglected for high SNR. We thus assume $\delta_{R}^{2}=0$ and $\delta_{b}^{2}=0$ for simplicity [20]. At the $\mathrm{U} 1$ and $\mathrm{U} 2$, the received signals can be written as

$$
y_{U 1}^{\mathrm{II}}=\sqrt{P_{R}} h_{R U 1} x_{R}+n_{U 1}, y_{U 2}^{\mathrm{II}}=\sqrt{P_{R}} h_{R U 2} x_{R}+n_{U 2} .
$$

The received SINR for $\mathrm{U} 1$ can be given by

$$
\gamma_{U 1}^{\mathrm{II}}=\frac{\alpha_{1} \beta \eta P_{B S}\left|h_{B S R}\right|^{2}\left|h_{R U 1}\right|^{2}}{\alpha_{2} \beta \eta P_{B S}\left|h_{B S R}\right|^{2}\left|h_{R U 1}\right|^{2}+\beta \eta \delta_{R}^{2}\left|h_{R U 1}\right|^{2}+\frac{\beta \eta \delta_{b}^{2}}{1-\beta}\left|h_{R U 1}\right|^{2}+\delta_{U 1}^{2}} .
$$

As previous operation of SIC at the U2, the U1's signal is detected first and then subtracts the desired signal of U2 from the residual signal. The corresponding SINRs for the U2 to successively detect two signals are respectively given by

$$
\gamma_{U 2,1}^{\mathrm{II}}=\frac{\alpha_{1} \beta \eta P_{B S}\left|h_{B S R}\right|^{2}\left|h_{R U 2}\right|^{2}}{\alpha_{2} \beta \eta P_{B S}\left|h_{B S R}\right|^{2}\left|h_{R U 2}\right|^{2}+\beta \eta \delta_{R}^{2}\left|h_{R U 2}\right|^{2}+\frac{\beta \eta \delta_{b}^{2}}{1-\beta}\left|h_{R U 2}\right|^{2}+\delta_{U 2}^{2}}
$$

and

$$
\gamma_{U 2,2}^{\text {II }}=\frac{\alpha_{2} \beta \eta P_{B S}\left|h_{B S R}\right|^{2}\left|h_{R U 2}\right|^{2}}{\beta \eta \delta_{R}^{2}\left|h_{R U 2}\right|^{2}+\frac{\beta \eta \delta_{b}^{2}}{1-\beta}\left|h_{R U 2}\right|^{2}+\delta_{U 2}^{2}} .
$$

\section{Outage Performance and Parameter Optimization}

\subsection{Outage Probability Analysis}

The outage probability is defined as the probability that the achievable rate of the receiver is less than the required threshold rate during a specified time block [21], which is the basis for calculating the performance indicators of communication systems, i.e., spectral efficiency and energy efficiency [22-24]. Therefore, we will focus on obtaining the closed-form solution of the system's outage probability in this subsection. According to the above analysis, an outage event occurs when the achievable rates for both users $\mathrm{U} 1$ and $\mathrm{U} 2$ are lower than the target data rate $r_{D}$. Based on the SIC process at the U2, 
the U1's signal is detected first in both half phases. Thus, the probability to characterize such an outage event for $\mathrm{U} 2$ can be formulated as

$$
P_{\text {out }}^{U 2}=\left(1-\operatorname{Pr}\left\{\gamma_{U 2,1}^{\mathrm{I}} \geq R_{D}, \gamma_{U 2,2}^{\mathrm{I}} \geq R_{D}\right\}\right)\left(1-\operatorname{Pr}\left\{\gamma_{U 2,1}^{\mathrm{II}} \geq R_{D}, \gamma_{U 2,2}^{\mathrm{II}} \geq R_{D}\right\}\right),
$$

where $R_{D}=2^{2 r_{D}}-1$. By substituting (6), (11), and (12) into (13), the outage probability of the U2 is given as the following proposition.

Proposition 1. Let $a=\alpha_{1} \beta \eta P_{B S}, b=\alpha_{2} \beta \eta P_{B S}, c=\beta \eta \delta_{R^{\prime}}^{2}$ and $d=\frac{\beta \eta \delta_{b}^{2}}{1-\beta}$. The outage probability of the $U 2$ is given by

- if $R_{D} \geq \frac{\alpha_{1}}{\alpha_{2}}$ or $R_{D}<\frac{\alpha_{1}-\alpha_{2}}{\alpha_{2}}$

$$
P_{\text {out }}^{U 2}=\left(1-\exp \left\{-\frac{R_{D} \delta_{U 2}^{2}}{\alpha \lambda_{B S U 2}}\right\}\right)\left(1-2 \exp \left\{-\frac{(c+d) R_{D}}{b \lambda_{B S R}}\right\} \sqrt{\frac{\delta_{U 2}^{2} R_{D}}{b \lambda_{B S R} \lambda_{R U 2}}} K_{1}\left(\sqrt{\frac{4 \delta_{U 2}^{2} R_{D}}{b \lambda_{B S R} \lambda_{R U 2}}}\right)\right\}
$$

where $K_{1}(\cdot)$ denotes the first order modified Bessel function with second kind [25].

- if $\frac{\alpha_{1}-\alpha_{2}}{\alpha_{2}} \leq R_{D}<\frac{\alpha_{1}}{\alpha_{2}}$

$$
\begin{aligned}
& P_{\text {out }}^{U 2}=\left(1-\exp \left\{-\frac{R_{D} \delta_{U 2}^{2}}{\left(a-b R_{D}\right) \lambda_{B S U 2} P_{B S}}\right\}\right)\left(1-2 \exp \left\{-\frac{(c+d) R_{D}}{\left(a-b R_{D}\right) \lambda_{B S R}}\right\} \sqrt{\frac{\delta_{U 2}^{2} R_{D}}{\left(a-b R_{D}\right) \lambda_{B S R} \lambda_{R U 2}}}\right. \\
& \left.\times K_{1}\left(\sqrt{\frac{4 \delta_{U 2}^{2} R_{D}}{\left(a-b R_{D}\right) \lambda_{B S R} \lambda_{R U 2}}}\right)\right) .
\end{aligned}
$$

Proof. Please refer to Appendix A.

For the U1, it will decode its desired signal $x_{1}$ by viewing the signal $x_{2}$ as interference during two half phases. Therefore, the outage probability of the U1 can be expressed as

$$
P_{\text {out }}^{U 1}=\operatorname{Pr}\left\{\max \left(\gamma_{U 1}^{\mathrm{I}}, \gamma_{U 1}^{\mathrm{II}}\right)<R_{D}\right\} .
$$

Then, the simplified mathematical expression of (16) is given by

- if $R_{D} \geq \frac{\alpha_{1}}{\alpha_{2}}$

$$
P_{\text {out }}^{U 1}=1
$$

- $\quad$ if $R_{D}<\frac{\alpha_{1}}{\alpha_{2}}$

$$
\begin{aligned}
& P_{\text {out }}^{U 1}=\left(1-\exp \left\{-\frac{R_{D} \delta_{U 1}^{2}}{\left(\alpha_{1}-\alpha_{2} R_{D}\right) \lambda_{B S U 1} P_{B S}}\right\}\right) \\
& \times\left(1-2 \exp \left(-\frac{(c+d) R_{D}}{\left(a-b R_{D}\right) \lambda_{B S R}}\right) \sqrt{\frac{\delta_{U 1}^{2} R_{D}}{\left(a-b R_{D}\right) \lambda_{R U 1} \lambda_{B S R}}} K_{1}\left(\sqrt{\frac{4 \delta_{U 1}^{2} R_{D}}{\left(a-b R_{D}\right) \lambda_{R U 1} \lambda_{B S R}}}\right)\right) .
\end{aligned}
$$

The derived result of $P_{o u t}^{U 1}$ is omitted because it is similar to Proposition 1.

\subsection{Parameters Joint Optimization}

In this work, the relay node $\mathrm{R}$ first harvests energy and then assists to forward the BS's signals. For power allocation coefficients $\alpha_{1}$ and $\alpha_{2}$, the NOMA users cannot achieve optimal reception at the same time. In addition, there also exists a trade-off between the transmit power of node $\mathrm{R}$ and residual signal for information transmission, where the higher proportion of information split $\beta$ can 
guarantee the node $\mathrm{R}$ to harvest more energy but less residual signal for information forwarding. Therefore, the optimal parameter such as $\alpha_{1}^{*}, \alpha_{2}^{*}$, and $\beta^{*}$ can be jointly obtained when each of user's performance metrics meets the required QoS. With the development of $5 \mathrm{G}$ communication systems, more and more devices will be connected to the network, which will increase the overall energy consumption. Therefore, from the perspective of green communications, the optimization of EE is particularly important. Based on the results of outage probabilities for both users U1 and U2, we aim to find the optimal parameters that can maximize the average energy efficiency $\omega_{E E}$ of the proposed system while protecting the achievable rate for each user. According to (13) and (16), the optimization problem can be equivalently expressed as (P1)

$$
\begin{array}{ll}
\max _{\alpha_{1}, \alpha_{2}, \beta} & \omega_{E E}=\frac{1}{P_{B S}} R_{D}\left(2-P_{\text {out }}^{U 1}-P_{\text {out }}^{U 2}\right) \\
\text { s.t. } \mathrm{C} 1: \gamma_{U}=\min \left\{\gamma_{U 1}^{\mathrm{I}}, \gamma_{U 1}^{\mathrm{II}}, \gamma_{U 2,2}^{\mathrm{I}}, \gamma_{U 2,2}^{\mathrm{II}}\right\} \geq R_{D} ; \\
\quad \mathrm{C} 2: 0<\alpha_{2}<\alpha_{1}<1, \alpha_{1}+\alpha_{2}=1 ; \\
\quad \mathrm{C} 3: 0<\beta<1 .
\end{array}
$$

In $\mathrm{C} 1, R_{D}>0$ illustrates the minimum SINR required for the U1 and U2. C2 and C3 denote the power allocation ratio constraint and information splitting proportion constraint, respectively.

Since the complex expressions of $P_{\text {out }}^{U 1}$ and $P_{\text {out }}^{U 2}$, the optimization problem (P1) cannot be solved analytically. However, it can be solved by a full-search algorithm, which will compute the key performance indicators of the proposed system with considered parameters. When the system achieves the maximum energy efficiency, the corresponding parameter values are the global optimal values. The detailed procedure is described in Algorithm 1.

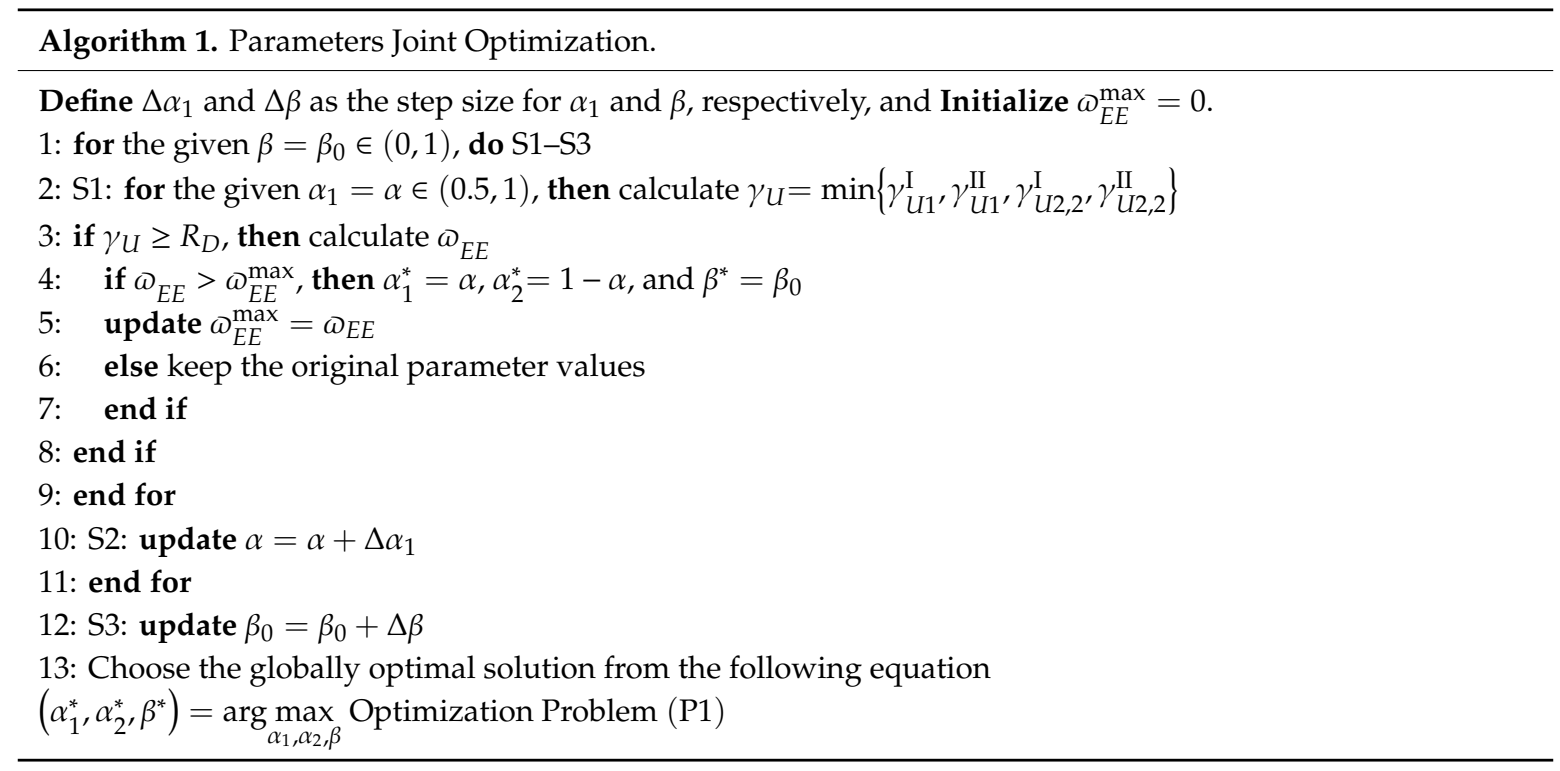

It should be noted that the reference [20] also analyzes the optimization problem of maximizing the whole achievable rate with constraints. By proving that the objective function satisfies the characteristics of the bi-convex optimization, the global optimal solution can be obtained by a proposed optimization algorithm, i.e., alternate convex search (ACS) algorithm [26], where only the variables of an active block are optimized while those of the other blocks are fixed. Moreover, the ACS algorithm can be guaranteed to converge to the partial optimum. In this paper, we consider the problem of maximizing the EE. Because the closed form of outage probabilities for both receivers is extremely complex, it is difficult to prove whether it satisfies the convex optimization. Therefore, in order to further improve the EE of the system, we will utilize the full-search algorithm to obtain the global optimal solution. Although the algorithm is more complex than the convex optimization algorithm, the search range is limited 
and the time to obtain the optimal value in actual simulation is not long. For the selection of optimal parameters, the algorithm complexity is $O\left(n^{2}\right)$ because two parameters need to be traversed jointly.

\section{Numerical Results}

In this section, the numerical results of the derived expressions are presented and the impact of key parameters on the performance of the considered system is discussed. In all simulations, we consider a scenario where the distances from BS to node $\mathrm{R}, \mathrm{U} 1$, and $\mathrm{U} 2$ are $5 \mathrm{~m}, 10 \mathrm{~m}$, and $7.5 \mathrm{~m}$, respectively, and distances from node $\mathrm{R}$ to $\mathrm{U} 1$ and $\mathrm{U} 2$ are $5 \mathrm{~m}$ and $2.5 \mathrm{~m}$, respectively. Path-loss exponent $\theta$ is set as $\theta=3$ for all channels. The energy conversion efficiency $\eta$ is 0.5 . For simplicity, we assume that the noise power of all receivers is the same, i.e., $\delta_{i}^{2}=-20 \mathrm{dBm}$.

Figure 2 depicts the outage probabilities for both the $\mathrm{U} 1$ and $\mathrm{U} 2$ under BS's transmit power $P_{B S}$ for different power allocation coefficients $\alpha_{1}$ and $\alpha_{2}$. We also compare the proposed spectrum sharing scheme with other schemes based on underlay CR networks with SWIPT [17] and direct transmission. For the decode-and-forward (DF) based spectrum sharing scheme [17], the secondary system can use the licensed spectrum with constraint transmit power. The direct transmission means the BS sends its signal to receivers without assisting from relay node. Figure $2 \mathrm{a}, \mathrm{b}$ shows that the outage performance of both users is improved with the increase of $P_{B S}$. In Figure 2a, when $\alpha_{1}=0.9$ and $\alpha_{2}=0.1$, the outage probability of $\mathrm{U} 1$ with proposed scheme will be better than that of direct transmission with the increase of $P_{B S}$, which is because that the higher transmit power may bring more interference for decoding. However, when $\alpha_{1}=0.8$ and $\alpha_{2}=0.2$, the outage probability of U1 with the proposed scheme always higher than that in the direct transmission, which is because that the allocated power coefficient for $\mathrm{U} 1$ is still 0.8 in the second phase and its transmission performance will be affected negatively. Obviously, the proposed spectrum sharing scheme outperforms the scheme in [17] within the range of selected parameters in terms of U1's outage probability. From Figure 2b, we can see that the outage performance of $\mathrm{U} 2$ under the proposed scheme is better than that of direct transmission in low $P_{B S}$ and then becomes worse with the increase of $P_{B S}$ for different values of $\alpha_{1}$ and $\alpha_{2}$. Compared with scheme in [17], the outage probability of $\mathrm{U} 2$ is lower when the proposed scheme is adopted for $\alpha_{1}=0.8$ and $\alpha_{2}=0.2$. However, when $\alpha_{1}=0.9$ and $\alpha_{2}=0.1$, the outage probability of U2 is only better than the scheme in [17] within a low range of $P_{B S}$. Moreover, the derived theoretical expressions of both the U1 and U2 agree well with simulation results, which indicates the outage performance analyses are validated.

In Figure 3, we investigate the outage probabilities for both the U1 and U2 with respect to $P_{B S}$ for different target rates $r_{D}$. As expect, the outage performance of both $\mathrm{U} 1$ and $\mathrm{U} 2$ is similar as Figure 2. Moreover, with the increase of target rates, the outage performance of both U1 and U2 are deteriorated because it is more difficult for transmission channels to support a higher rate requirement. As shown in Figures 2 and 3, when the proposed scheme is adopted, the outage performances of U1 and $\mathrm{U} 2$ are better than that of direct transmission in the high and low value ranges of $P_{B S}$, respectively. Furthermore, it can be seen that the proposed scheme is always superior to the scheme in [17] within the set value range in terms of outage probabilities of U1 and U2. The exact analytical results are matched well with simulation results.

Figure 4 plots the average energy efficient improvement ratio of the proposed scheme compared with the direct transmission without spectrum sharing and scheme in [17]. The proposed parameters joint optimization algorithm is also employed in this simulation. As shown in Figure 4, the whole energy efficient improvement ratio of the proposed scheme is deteriorated with increase of $P_{B S}$ since, although the higher BS's transmit power can improve the achievable rate, it cannot compensate for the loss of performance due to power consumption. Even in this case, the overall energy efficient improvement ratio remains above $100 \%$ when compared with direct transmission, while the overall energy efficiency is also improved compared with the scheme in [17] when $P_{B S}$ is in low range. Moreover, the higher target transmission rate $r_{D}$ will result in a higher energy efficient improvement ratio when $P_{B S}$ are less than $-5 \mathrm{~dB}$ and $1 \mathrm{~dB}$, respectively, because, although it is more difficult for the 
channel to support a higher rate requirement, the achievable rate can be still improved. Therefore, the overall energy efficiency will be increased due to the lower consumption of power. On the contrary, when $P_{B S}$ are greater than $-5 \mathrm{~dB}$ and $1 \mathrm{~dB}$, respectively, the ratio of the energy efficiency with the proposed scheme to the energy efficiencies of direct transmission and scheme in [17] are approximately 2 and 1, respectively. Therefore, the improvement ratio of the energy efficiencies remains around $100 \%$ and $0 \%$, respectively. It can be seen from the simulation results that the proposed scheme can effectively improve the overall energy efficiency of the system in the whole range of transmit power $P_{B S}$ when compared with direct transmission, while the proposed scheme still has obvious advantages over the scheme in [17] in terms of energy efficiency.

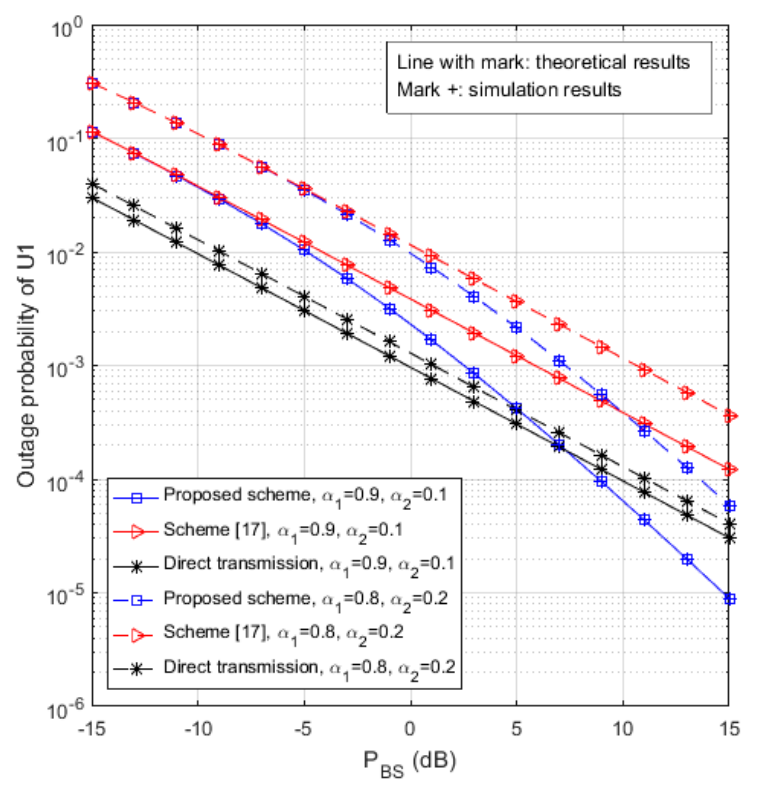

(a)

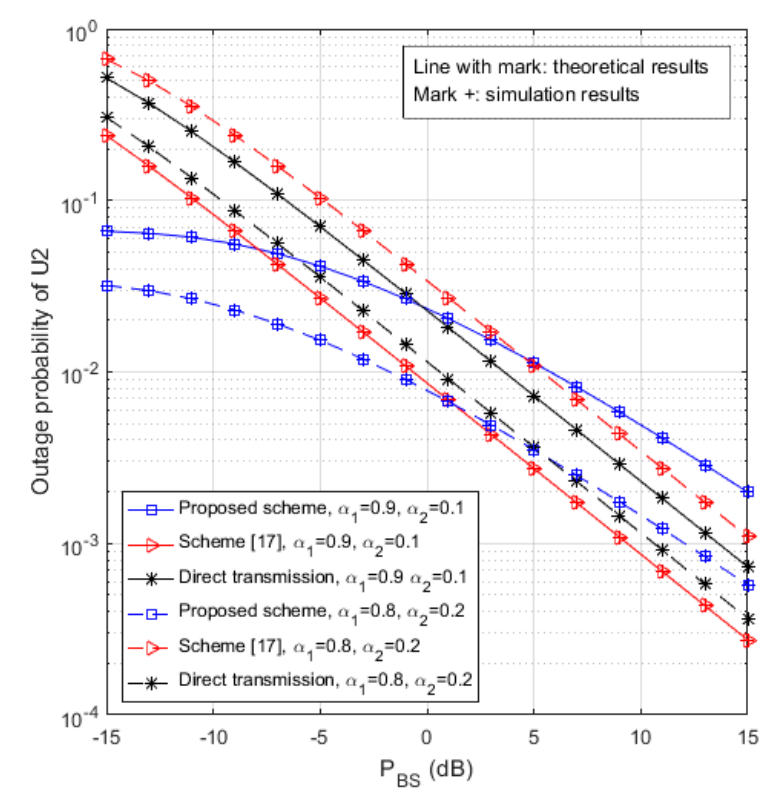

(b)

Figure 2. Outage probabilities of U1 (a) and U2 (b) w.r.t $P_{B S}$ for different $\alpha_{1}$ and $\alpha_{2} . \quad \beta=0.5$, $r_{D}=1.0 \mathrm{bit} / \mathrm{s} / \mathrm{Hz}$. 


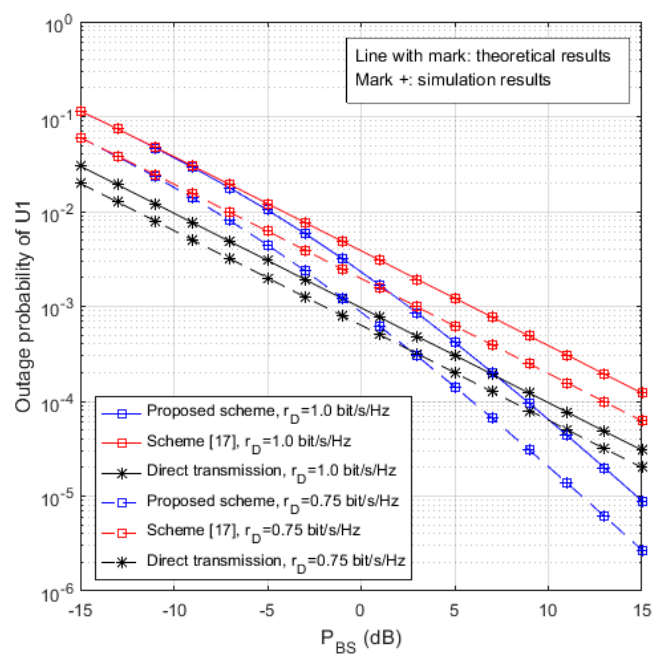

(a)

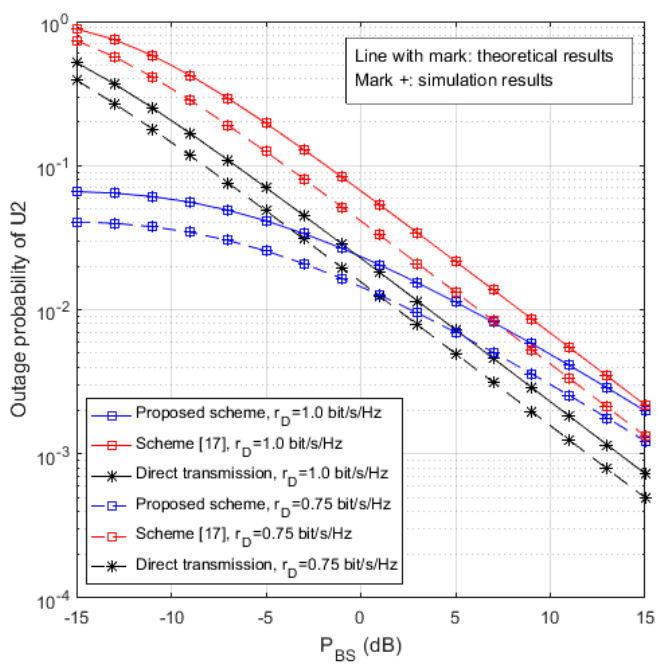

(b)

Figure 3. Outage probabilities of $\mathrm{U} 1$ (a) and $\mathrm{U} 2$ (b) w.r.t $P_{B S}$ for different $r_{D} \cdot \alpha_{1}=0.9, \alpha_{2}=0.1, \beta=0.5$.

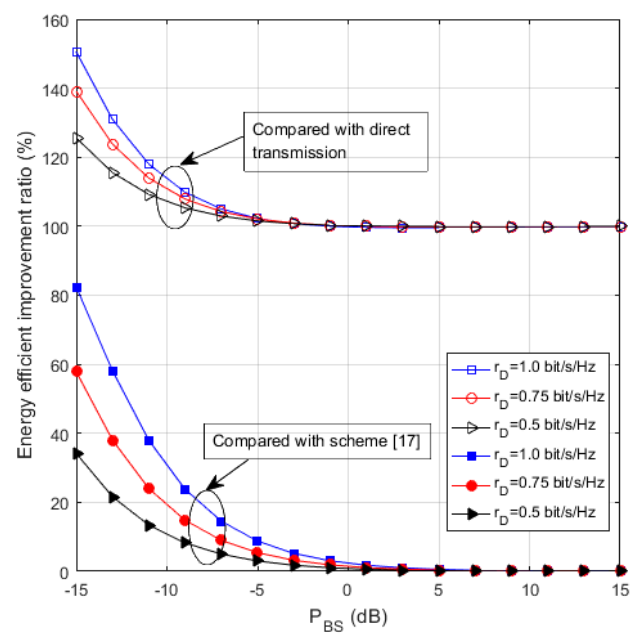

Figure 4. Total energy efficient improvement ratio compared with direct transmission w.r.t $P_{B S}$ for different $r_{D}$. 


\section{Conclusions}

In this paper, we have proposed a relay-assisted spectrum sharing scheme by adopting AF technique with SWIPT in a NOMA-based cognitive radio network. Analytical expressions of outage probabilities for both receivers U1 and U2 were derived exactly. Analytical deductions were validated via numerical simulations. In order to obtain the optimal value of parameters, we proposed a joint parameter optimization algorithm to maximize the average energy efficiency under required transmission rates. The simulation results revealed that the proposed optimization algorithm for spectrum sharing scheme can provide significant performance improvement for considered system in terms of energy efficiency. For the number of users in this system model, we consider two users as an example, aiming to verify the availability of the proposed spectrum sharing scheme with optimal parameter allocations. Next, we will investigate more complex system model, such as several relay nodes, first to harvest energy and then assist to forward signals of $N$ users, which are clustered with a game algorithm in order to achieve the maximal system achievable rate.

Author Contributions: K.T. wrote the original manuscript and simulated numerical results, spell-checked, and verified this paper; S.L. provided the idea of this paper and revised this paper. All authors have read and agreed to the published version of the manuscript.

Funding: This work was supported by the National Natural Science Foundation of China under Grant No. 61931009, in part by the Research Foundation of China Postdoctoral Science Foundation under Grant No. 2019M652895, in part by the Research Foundation of Education Department of Hunan Province under No. 18 B517.

Conflicts of Interest: The authors declare no conflict of interest.

\section{Appendix A}

Let $X=\left|h_{B S U 2}\right|^{2}, Y=\left|h_{B S R}\right|^{2}$, and $Z=\left|h_{R U 2}\right|^{2}$, Equation (12) can be rewritten as

$$
\begin{aligned}
& P_{\text {out }}^{U 2}=\left(1-\operatorname{Pr}\left\{X \geq \frac{R_{D} \delta_{S U 2}^{2}}{\alpha_{1} P_{B S}-\alpha_{2} P_{B S} R_{D}}, X \geq \frac{R_{D} \delta_{S U 2}^{2}}{\alpha_{2} P_{B S}}\right\}\right) \\
& \times\left(1-\operatorname{Pr}\left\{Y \geq \frac{(c+d) R_{D}}{a-b R_{D}}+\frac{\delta_{S U 2}^{2} R_{D}}{\left(a-b R_{D}\right) Z}, Y \geq \frac{(c+d) R_{D}}{b}+\frac{\delta_{S U 2}^{2} R_{D}}{b Z}\right\}\right) .
\end{aligned}
$$

For the first term of (A1), when $\alpha_{2} P_{B S} \geq \alpha_{1} P_{B S}-\alpha_{2} P_{B S} R_{D}>0$, we have $\frac{\alpha_{1}-\alpha_{2}}{\alpha_{2}} \leq R_{D}<\frac{\alpha_{1}}{\alpha_{2}}$, thus the data range of $X$ is $X \geq \frac{R_{D} \delta_{S L 2}^{2}}{\alpha_{1} P_{B S}-\alpha_{2} P_{B S} R_{D}}$. When $\alpha_{2} P_{B S}<\alpha_{1} P_{B S}-\alpha_{2} P_{B S} R_{D}$, we have $R_{D}<\frac{\alpha_{1}-\alpha_{2}}{\alpha_{2}}$, the data range of $X$ is $X \geq \frac{R_{D} \delta_{S U 2}^{2}}{\alpha_{2} P_{B S}}$. Furthermore, when $\alpha_{1} P_{B S}-\alpha_{2} P_{B S} R_{D} \leq 0$, we can obtain $R_{D} \geq \frac{\alpha_{1}}{\alpha_{2}}$ with $X \geq \frac{R_{D} \delta_{S L 2}^{2}}{\alpha_{2} P_{B S}}$. To sum up, the value rang of $X$ can be concluded as

$$
X \geq\left\{\begin{array}{l}
\frac{R_{D} \delta_{S U 2}^{2}}{\alpha_{2} P_{B S}}, \text { if } R_{D} \geq \frac{\alpha_{1}}{\alpha_{2}} \text { or } R_{D}<\frac{\alpha_{1}-\alpha_{2}}{\alpha_{2}} \\
\frac{R_{D} \delta_{S U 2}^{2}}{\alpha_{1} P_{B S}-\alpha_{2} P_{B S} R_{D}}, \text { if } \frac{\alpha_{1}-\alpha_{2}}{\alpha_{2}} \leq R_{D}<\frac{\alpha_{1}}{\alpha_{2}}
\end{array}\right.
$$

Similar to the above analysis, for the second term of (A1), the value range for $Y$ is given by

$$
Y \geq\left\{\begin{array}{c}
\frac{(c+d) R_{D}}{b}+\frac{\delta_{S U 2}^{2} R_{D}}{b Z}, \text { if } R_{D} \geq \frac{\alpha_{1}}{\alpha_{2}} \text { or } R_{D}<\frac{\alpha_{1}-\alpha_{2}}{\alpha_{2}} \\
\frac{(c+d) R_{D}}{a-b R_{D}}+\frac{\delta_{S U 2}^{2} R_{D}}{\left(a-b R_{D}\right) Z}, \text { if } \frac{\alpha_{1}-\alpha_{2}}{\alpha_{2}} \leq R_{D}<\frac{\alpha_{1}}{\alpha_{2}}
\end{array}\right.
$$

For the exponentially distributed random variables $X, Y$, and $Z$, Equation (12) can be generally written as

- if $R_{D} \geq \frac{\alpha_{1}}{\alpha_{2}}$ or $R_{D}<\frac{\alpha_{1}-\alpha_{2}}{\alpha_{2}}$

$$
P_{\text {out }}^{U 2}=\left(1-\int_{\frac{R_{D} \delta_{S U 2}^{2}}{\alpha_{2} P_{B S}}}^{\infty} \frac{1}{\lambda_{B S U 2}} \exp \left(-\frac{x}{\lambda_{B S U 2}}\right) d x\right)\left(1-\int_{0}^{\infty} \int_{\frac{(c+d) R_{D}}{b}+\frac{\delta_{S U 2}^{2} R_{D}}{b z}}^{\infty} \frac{1}{\lambda_{B S R} \lambda_{R U 2}} \exp \left(-\frac{y}{\lambda_{B S R}}-\frac{z}{\lambda_{R U 2}}\right) d y d z\right) .
$$


- $\quad$ if $\frac{\alpha_{1}-\alpha_{2}}{\alpha_{2}} \leq R_{D}<\frac{\alpha_{1}}{\alpha_{2}}$

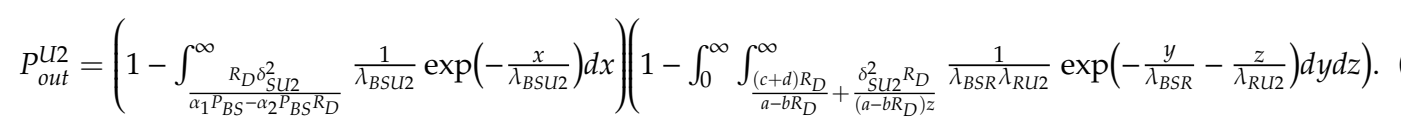

After some mathematical operations, the closed-form expression of (12) can be calculated as (13) and (14) for different values of $R_{D}$.

\section{References}

1. Luo, S.; Teh, K.C. Adaptive Transmission for Cooperative NOMA System with Buffer-Aided Relaying. IEEE Commun. Lett. 2017, 4, 937-940. [CrossRef]

2. Qin, Z.; Yue, X.; Liu, Y.; Ding, Z.; Nallanathan, A. User Association and Resource Allocation in Unified NOMA Enabled Heterogeneous Ultra-Dense Networks. IEEE Commun. Mag. 2018, 56, 86-92. [CrossRef]

3. Zhang, J.; Tao, X.; Wu, H.; Zhang, X. Performance Analysis of Users Pairing in Cooperative NOMA Networks. IEEE Access 2018, 6, 74288-74302. [CrossRef]

4. Zhai, C.; Zhang, W.; Mao, G. Cooperative Spectrum Sharing Between Cellular and Ad-Hoc Networks. IEEE Trans. Wirel. Commun. 2014, 13, 4025-4037. [CrossRef]

5. Liang, W.; Ng, S.X.; Hanzo, L. Cooperative Overlay Spectrum Access in Cognitive Radio Networks. IEEE Commun. Surv. Tutor. 2017, 19, 1924-1944. [CrossRef]

6. Zhang, H.; Guo, Y.X.; Zhong, Z.; Wu, W. Cooperative Integration of RF Energy Harvesting and Dedicated WPT for Wireless Sensor Networks. IEEE Microw. Wirel. Compon. Lett. 2019, 29, 291-293. [CrossRef]

7. Liu, Z.; Lu, G.; Ye, Y.; Shi, L. On the Performance of Battery-Assisted PS-SWIPT Enabled DF Relaying. Information 2020, 11, 165. [CrossRef]

8. Lv, L.; Chen, J.; Ni, Q. Cooperative Non-Orthogonal Multiple Access in Cognitive Radio. IEEE Commun. Lett. 2016, 20, 2059-2062. [CrossRef]

9. Xu, W.; Li, X.; Lee, C.H.; Pan, M.; Feng, Z. Joint Sensing Duration Adaption, User Matching, and Power Allocation for Cognitive OFDM-NOMA Systems. IEEE Trans. Wirel. Commun. 2018, 17, 1269-1282. [CrossRef]

10. Arzykulov, S.; Nauryzbayev, G.; Tsiftsis, T.A.; Maham, B. Performance Analysis of Underlay Cognitive Nonorthogonal Multiple Access Networks. IEEE Trans. Veh. Technol. 2019, 68, 9318-9322. [CrossRef]

11. Li, B.; Qi, X.; Huang, K.; Fei, Z.; Zhou, F.; Hu, Q. Security-Reliability Tradeoff Analysis for Cooperative NOMA in Cognitive Radio Networks. IEEE Commun. Lett. 2019, 67, 83-96. [CrossRef]

12. Hedayati, M.; Kim, I.-M. On the Performance of NOMA in the Two-User SWIPT System. IEEE Trans. Veh. Technol. 2018, 67, 11258-11263. [CrossRef]

13. Xu, Y.; Shen, C.; Ding, Z.; Sun, X.; Yan, S.; Zhu, G.; Zhong, Z. Joint Beamforming and Power-Splitting Control in Downlink Cooperative SWIPT NOMA Systems. IEEE Trans. Signal Process. 2017, 65, 4874-4886. [CrossRef]

14. Bariah, L.; Muhaidat, S.; A-Dweik, A. Error Probability Analysis of NOMA-based Relay Networks with SWIPT. IEEE Commun. Lett. 2019, 23, 1223-1226. [CrossRef]

15. Zhang, Y.; Yang, Q.; Zheng, T.X.; Wang, H.; Ju, Y.; Meng, Y. Energy Efficiency Optimization in Cognitive Radio Inspired Non-Orthogonal Multiple Access. In Proceedings of the IEEE 27th International Symposium on Personal, Indoor and Mobile Radio Communications PIMRC, Valencia, Spain, 4-8 September 2016.

16. Wang, X.; Na, Z.; Lam, K.Y.; Liu, X.; Wang, L. Energy Efficiency Optimization for NOMA-based Cognitive Radio with Energy Harvesting. IEEE Access 2019, 7, 139172-139180. [CrossRef]

17. Yu, Y.; Yang, Z.; Wu, Y.; Hussein, J.A.; Jia, W.K.; Dong, Z. Outage Performance of NOMA in Cooperative Cognitive Radio Networks with SWIPT. IEEE Access 2019, 7, 117308-117317. [CrossRef]

18. Tang, K.; Shi, R.; Dong, J. Throughput Analysis of Cognitive Wireless Acoustic Sensor Networks with Energy Harvesting. Future Gener. Comput. Syst. 2018, 86, 1218-1227. [CrossRef]

19. Zhai, C.; Liu, J.; Zheng, L. Relay-Based Spectrum Sharing with Secondary Users Powered by Wireless Energy Harvesting. IEEE Commun. Lett. 2016, 64, 1875-1887.

20. Wang, Z.; Chen, Z.; Xia, B.; Luo, L.; Zhou, J. Cognitive Relay Networks with Energy Harvesting and Information Transfer: Design, Analysis, and Optimization. IEEE Trans. Wirel. Commun. 2016, 15, 2562-2576. [CrossRef] 
21. Hieu, T.C.; Cuong, N.L.; Hoang, T.M.; Quan, D.T.; Hiep, P.T. On Outage Probability and Ergodic Rate of Downlink Multi-User Relay Systems with Combination of NOMA, SWIPT, and Beamforming. Sensors 2020, 20, 4737. [CrossRef]

22. Nguyen, H.V.; Kim, H.M.; Kang, G.M.; Nguyen, K.H.; Bui, V.P.; Shin, O.S. A Survey on Non-Orthogonal Multiple Access: From the Perspective of Spectral Efficiency and Energy Efficiency. Energies 2020, 13, 4160. [CrossRef]

23. Zhang, Y.; Wang, X.; Wang, D.; Lan, Y.; Zhang, Y. Outage Performance of Relay Sparse Code Multiple Access Networks. In Proceedings of the 2019 IEEE Wireless Communications and Networking Conference (WCNC), Marrakesh, Morocco, 15-18 April 2019; pp. 1-6.

24. Tseng, S.; Tsai, C.; Yu, C. Outage-Capacity-Based Cross Layer Resource Management for Downlink NOMA-OFDMA Video Communications: Non-Deep Learning and Deep Learning Approaches. IEEE Access 2020, 8, 140097-140107. [CrossRef]

25. Gradshteyn, I.S.; Ryzhik, I.M. Table of Integrals, Series, and Products, 7th ed.; Academic Press: New York, NY, USA, 2007.

26. Gorski, J.; Pfeuffer, F.; Klamroth, K. Biconvex Sets and Optimization with Biconvex Functions: A Survey and Extensions. Math. Methods Oper. Res. 2007, 66, 373-407. [CrossRef]

Publisher's Note: MDPI stays neutral with regard to jurisdictional claims in published maps and institutional affiliations.

(C) 2020 by the authors. Licensee MDPI, Basel, Switzerland. This article is an open access article distributed under the terms and conditions of the Creative Commons Attribution (CC BY) license (http://creativecommons.org/licenses/by/4.0/). 